\title{
Comparative study of cellulose fragmentation by enzymes and ultrasound
}

\author{
F. M. Gama, ${ }^{*}$ M. G. Carvalho, ${ }^{\dagger}$ M. M. Figueiredo, ${ }^{\dagger}$ and M. Mota* \\ ${ }^{*}$ Department of Biological Engineering, University of Minho, Braga, Portugal, and ${ }^{\dagger}$ Department \\ of Chemical Engineering, Coimbra University, Coimbra, Portugal
}

\begin{abstract}
The stability in aqueous suspensions of two particulate celluloses, Sigmacell type 100 and Avicel PH101, was analyzed. The effect of the presence of a cellulase from Trichoderma reesei, ionic strength, and ultrasonic agitation on the fragmentation/aggregation phenomena was studied.

Particle size distributions of the powders were obtained with three different particle sizers: the Galai CIS 100, the Coulter Multisizer II, and the Malver 2600c. The differences in the obtained absolute values are discussed. according to the measuring principles of each technique; however, the overall conclusions are independent of the particle sizer used. The enzyme breaks up the Avicel aggregates more effectively than ultrasound while the Sigmacell particles are stable under the present experimental conditions.

The stabilizing effect of cellulases was tentatively explained using the DLVO (Derjaguin, Landau, Verweye, and Overbeek) theory. The adsarbed enzyme did not change significantly the zeta potential of the fibers; hence, the stabilizing effect was attributed to a reduction in the attractive van der Waals forces and hydration effects. (C) 1997 by Elsevier Science Inc.
\end{abstract}

Keywords: Cellulose; size analysis; particle size distribution; cellulase; fragmentation

\section{Introduction}

Cellulose powders used as pharmaceutical excipients are widely utilized in the study of cellulolytic systems; however, some of their properties are difficult to characterize because of their irregular geometry and size polydispersity. Two types of particulate cellulose are recognized. One is the microcrystalline cellulose (e.g., Avicel PH101) which is a partially hydrolyzed cellulose prepared by heat-treating $\alpha-$ cellulose from wood with strong mineral acids, vigorously agitating the slurry, and spray drying. The other is the powdered cellulose (e.g., Sigmacell type 100) obtained by mechanically disintegrating purified $\alpha$-cellulose (dry or wet grinding and drying) generally from wood containing both broken fibers and irregularly shaped particles. The mechanical treatment results in partial amorphous character and limited depolymerization of cellulose. ${ }^{1,2}$

When placed in water, like virtually all materials, cellu-

Address reprint requests to M. J. M. Gomes Mota, Professor, Department of Biological Engineering, University of Minho, Largo do Paco, 4709 Braga codex, Portugal

Received 9 August 1995; accepted 28 February 1996 lose becomes electrically charged due to ionization of the carboxyl groups on the surface and specific adsorption of ions from solution. The resulting charges on the surface of the material induce a redistribution of the mobile ions in the solution and an electrical double layer is produced. The mutual interaction of the electrical double layers of two approaching particles originate repulsive and attractive forces-Coulombic and van der Walls, respectively. The strength of this interaction might also be influenced by steric effects and the existence of a hydration layer. ${ }^{3}$

The understanding of the electrokinetic properties of cellulosic materials may provide important clues for textile and papermaking industries, namely, to control the retention of pigments and dyes and drainage and flocculation processes. ${ }^{4}$ The implication of cellulases, xylanases, and other enzymes on the electrokinetic and aggregation properties of the cellulose fibers is also of relevant interest. ${ }^{5-8}$ Several researchers have reported a fragmentation effect of cellulases on cottons, microcrystalline celluloses, and pulps. ${ }^{6,9-15}$

The aim of the present work is to characterize the Avicel and Sigmacell particles and study their stabilization by cellulases. The techniques adopted to investigate the effect of cellulase were particle sizing and electrophoretic mobility. The latter was used to allow the interpretation of the results 
in terms of the DLVO (Derjaguin, Landau, Verweye, and Overbeek) theory. Additionally, sedimentation tests followed by optical density attenuation were performed in order to detect the formation of aggregates.

Regarding particle sizing, several techniques have been used to measure the particle size distribution of microcrystalline cellulose. Rather different values have been reported in the literature. The discrepancies mainly are attributed to the irregularity of the cellulose particles and their tendency to agglomerate. ${ }^{16}$ In this work, a comparative study will be performed with and without previous ultrasonic agitation using different particle sizers.

\section{Materials and methods}

\section{Materials}

The cellulase from Trichoderma reesei Multifect L-250 was a kind gift from Finnish Sugar Co., Ltd., Finland. Two types of cellulose were used: Sigmacell type 100 and Avicel PH101. The crystallinity, surface area, degree of polymerization, and porosity of these celluloses were characterized in previous works. ${ }^{17.18}$

\section{Enzymatic hydrolysis}

The reaction mixture containing $50 \mathrm{mg}$ of each kind of cellulose, $3.0 \mathrm{ml}$ of $50 \mathrm{~mm}$ acetate buffer $\mathrm{pH} 5.0$, and $1.0 \mathrm{ml}$ of diluted enzyme (1:100) was placed in a $50-\mathrm{ml}$ flask and incubated at $50^{\circ} \mathrm{C}$ in an end-to-end shaker for $0.5,12$, and $36 \mathrm{~h}$. The reaction medium was then centrifuged $(5,000 \mathrm{rpm}$ for $3 \mathrm{~min})$, the fibers were washed twice with distilled water, and resuspended in the same initial volume. The particle size distribution, optical density attenuation, and electrophoretic mobility were then analyzed as described below

\section{Particle size characterization}

The particle size distribution of the samples was first measured using the Galai CIS 100. This is a recently developed laser instrument based on the time of transition theory ${ }^{19}$ where the size of each individual particle is calculated from the time of interaction between the particles and the laser beam. The particles were suspended in a magnetically stirred cuvette and the size range was scanned from $0.5-150 \mu \mathrm{m}$.

In order to compare the results obtained and also to study the influence of the sizing technique, the size distribution was measured with a Coulter Multisizer II based on the Coulter principle ${ }^{20}$ and a laser diffractometer, the Malvern $2600 \mathrm{c}$. ${ }^{21}$

Since the operation principle on which the Coulter Multisizer is based required the use of an electrolyte, this was adopted as the suspending medium for the remaining techniques.

Samples were previously diluted, sonicated when mentioned ( 5 $\mathrm{min}$ ), and kept under magnetic agitation. From these stock suspensions, aliquots were withdrawn for the various particle sizers until the concentration level required for each technique was achieved. It should be mentioned that these values are dependent on the instrument utilized.

The Coulter Multisizer was calibrated with polymer latex spheres of $13.5 \mu \mathrm{m}$ nominal size (supplied by Coulter Electronics). The aperature sizes used were 100 and $140 \mu \mathrm{m}$ (the latter was utilized to characterize Avicel without ultrasonic agitation). The electrolyte was $0.9 \%(\mathrm{w} / \mathrm{v}) \mathrm{NaCl}$ aqueous solution (commercially known as ISOTON). The instrument was operated in the siphon mode with a constant sampled volume of $500 \mu \mathrm{l}$. Both the Galai and Malvern do not require calibration.

In the experiments with the Malvern (and similarly with the Galai), the suspension under analysis was contained in a small cell which was magnetically agitated. The resultant scattering pattern was converted to a particle size distribution using the Fraunhofer diffraction theory. ${ }^{21}$ The inversion algorithm was implemented in the system software. All the measurements were performed with a $100 \mathrm{~mm}$ focal length giving a nominal range of $1.9-188 \mu \mathrm{m}$.

\section{Electrophoretic mobility measurements}

The electrophoretic mobility of cellulose particles in aqueous suspension was measured in a Zeiss Zeta-Meter $3.0+$ by applying a potential of $300 \mathrm{~V}$ across the electrophoresis cell. The measured electrophoretic mobility allows the calculation of the zeta potential according to the equation of Helmholtz-Smoluchowski. ${ }^{22}$

Avicel and Sigmacell celluloses were submitted to ultrasonic agitation or enzymatic digestion during $0.5 \mathrm{~h}$ as described. These suspensions were prepared with a cellulose concentration of 0.5 $\mathrm{mg} / \mathrm{ml}$ in distilled water or $0.01 \mathrm{M} \mathrm{NaCl}$. The $\mathrm{pH}$ varied between $2.5-10.0$ using $\mathrm{HCl}$ or $\mathrm{NaOH}$. Samples were allowed to equilibrate for about $1 \mathrm{~h}$ in an orbital shaker.

\section{Optical density measurements}

Suspensions of each type of cellulose were prepared in order to obtain an initial optical density of 1.0 at $400 \mathrm{~nm}$. Some of these samples were then treated by ultrasonic agitation or enzymatic digestion which resulted in a higher optical density. In the latter case, the particles were washed as described previously and resuspended in the same volume of distilled water. In order to study the effect of the ionic strength, additional experiments using the sonicated samples were conducted at $0.05 \mathrm{M} \mathrm{NaCl}$ solution. These samples $(2 \mathrm{ml})$ were transferred to a glass cuvette and the optical density was recorded as a function of time using a Jasco 7850 spectrophotometer.

\section{DLVO calculations}

In order to explain the enzymatic fragmentation of the Avicel particles, the DLVO theory of colloid stability ${ }^{3}$ was used. The van der Waals attraction energy $\left(V_{\mathrm{A}}\right)$ and the energy of electrostatic interaction $\left(V_{\mathrm{R}}\right)$ were calculated by

$$
V_{\mathrm{A}}=-\left(A_{11} \cdot \mathrm{a}\right) /(12 \cdot \mathrm{d})
$$

and

$$
V_{\mathrm{R}}=2 \pi \epsilon \mathrm{a} \zeta^{2} e^{-\mathrm{kd}}
$$

where $a$ is the particle radius and $d$ is the distance between the particles surface; $\zeta$ is the zeta potential; $\epsilon$ is the permittivity of the solution; $\kappa$ is the Debye-Huckel parameter which depends on the ionic strength of the solution; and $A_{11}$ is the Hamaker constant for the interacting media. Two extreme cases were considered in the calculations which used either the Hamaker constant

$$
A_{11}=4.0 \times 10^{-20} \mathrm{~J}
$$

for cellulose particles interacting across water or

$$
A_{11}=0
$$

which corresponds to the absence of van der Walls forces between the hydrated protein layers. ${ }^{3,23}$

This methodology is valid for spherical particles. It represents, for the present case, an oversimplification; however, it should be noted that the cellulose particle size measured by any of the utilized sizers is expressed in terms of a spherical equivalent diameter which therefore had to be used in the calculations. 


\section{Results and discussion}

\section{Particle size characterization}

Particle size distribution was measured with the three particle sizers mentioned earlier for both Avicel and Sigmacell before and after enzymatic treatment. In the former case, mcasurements were performed with and without ultrasonic agitation which was denoted by nontreated (NT) and sonicated (US). The enzymatically treated samples were analyzed without previous sonication since this could mask the enzyme effect. Several incubation times were tested. Samples were designated by Ez1, Ez2, or Ez3 which corresponded to $0.5,12$, and $36 \mathrm{~h}$ of digestion, respectively.

The results presented in Table $l$ are in terms of the median size $\left(d_{50}\right)$ of volume distributions. Each value is an average of at least five independent measurements.

The results obtained for the NT and US samples (Table $I$ and Figure 1 ) suggest the following remarks:

The Sigmacell particles are clearly smaller than those of Avicel;

The values of $d_{50}$ are, in general, dependent on the measuring technique although they are within the same order of magnitude. The Malvern always gives the largest values and the Galai shows the lowest reproductivity;

Regarding the effect of ultrasonic agitation (Figure 1), the size reduction is much more evident for Avicel (about $50 \%$ ). Although the curves obtained with the other two techniques do not overlap, they exhibit the same trend;

It is also of interest to mention that the US Avicel sample presented a bimodal histogram whereas that of the NT sample was unimodal.

From these results, it can be concluded that direct comparisons of the size distributions obtained with the various instruments are not possible. This was somehow expected, since each technique measures a different particle parameter which for nonspherical particles (like those of cellulose), may lead to different results. ${ }^{24}$ Indeed, while the Coulter measures the particle volume and expresses the results as equivalent volume diameter, the Galai measures the average length of the particles crossing the laser beam at various angles. On the other hand, the Malvern produces a size

Table 1 Median sizes ( $d_{50}$ in $\mu \mathrm{m}$, on a volume basis) obtained for the various samples with different particle sizers

Particle sizer

\begin{tabular}{lrrrr}
\cline { 3 - 4 } Sample & \multicolumn{1}{c}{ Galai } & Malvern & Coulter \\
\hline \multirow{4}{*}{ Sigmacell } & NT & $10.1 \pm 0.7$ & $15.1 \pm 0.1$ & $10.9 \pm 0.5$ \\
& US & $9.4 \pm 0.5$ & $14.8 \pm 0.1$ & $10.4 \pm 0.5$ \\
& Ez1 & $9.5 \pm 0.3$ & $15.1 \pm 0.1$ & $11.2 \pm 0.2$ \\
& Ez3 & $10.3 \pm 0.4$ & $16.7 \pm 0.1$ & $12.3 \pm 0.3$ \\
& NT & $13.9 \pm 0.6$ & $21.5 \pm 0.2$ & $16.1 \pm 0.6$ \\
Avicel & US & $20.6 \pm 2.4$ & $51.4 \pm 0.2$ & $30.6 \pm 1.1$ \\
& E21 & $16.8 \pm 3.0$ & $26.3 \pm 0.1$ & $15.6 \pm 0.8$ \\
& Ez2 & $20.0 \pm 3.8$ & $23.5 \pm 0.1$ & $14.7 \pm 0.6$ \\
& Ez3 & $22.0 \pm 4.1$ & $27.5 \pm 0.2$ & $17.3 \pm 0.4$ \\
& & & & $19.7 \pm 0.9$ \\
\hline
\end{tabular}

distribution which is suppose to represent a volume distribution of the projected area diameter in random orientation. $^{25}$

The sample handling system of the instruments used are also different. As stated before, a magnetically stirred small cell was used to hold the suspension in the Galai and Malvern whereas in the Coulter Multisizer, a totally different configuration was employed. In the Coulter, the particles were suspended in a mechanically agitated beaker $(200-\mathrm{ml})$ and forced to flow through a small orifice. This arrangement could eventually break agglomerates that might exist in the Avicel sample (not previously sonicated) which were not destroyed by the magnetic stirring used in the remaining techniques. This would explain, at least partially, the low values obtained with the Coulter. Besides, the meaning of an equivalent volume diameter applied to fibers may also be questionable.

Nonetheless, the values obtained here for the Avicel are comparable to those reported in the literature for the median Stokes diameter. ${ }^{15,26}$ These vary from $46-54 \mu \mathrm{m}$ for agglomerated cellulose and 23-29 $\mu \mathrm{m}$ after sonication. It should be mentioned once again that these values were obtained with another technique (photosedimentation) and therefore a perfect coincidence would not be expected.

Regarding the results obtained after enzymatic treatment, as can be seen in Table 1, the following comments can be made:

Comparing the US and Ezl samples, it is clear that for Sigmacell, the median values $\left(d_{50}\right)$ are quite close. Higher deviations are, however, detected for the correspondent Avicel samples;

As far as incubation time is concerned, Table 1 indicates that longer digestion periods lead to larger particle sizes. Figure 2 illustrates these differences in terms of cumulative size distributions (obtained with the Galai) for various digestion times. As can be seen, the cumulative curves are shifted to the right as the digestion periods increase. Exactly the same tendency was found with the other particle sizers;

For Avicel and similar to the behavior of the US sample, the enzymatically treated samples also presented a bimodal distribution. For longer digestion periods, this profile tends to become unimodal (like the NT samples).

Concerning fragmentation, it can thus be concluded especially for Avicel that the effect of ultrasound is similar to that of the enzymes for short incubation intervals. The forces that keep the Avicel particles aggregated must therefore be of a physical nature (since ultrasound is not expected to break covalent bonds). On the other hand, the larger median values obtained for longer digestion periods for both Avicel and Sigmacell can be explained by the conversion of cellulose to soluble sugars where the smaller particles were hydrolyzed faster. These results will be explained in a forthcoming publication. Furthermore, it should be emphasized that the results of Table $I$ also reveal that the percent increase in the median values of these samples using Ez1 as reference is the same independently of the sizing technique used. 


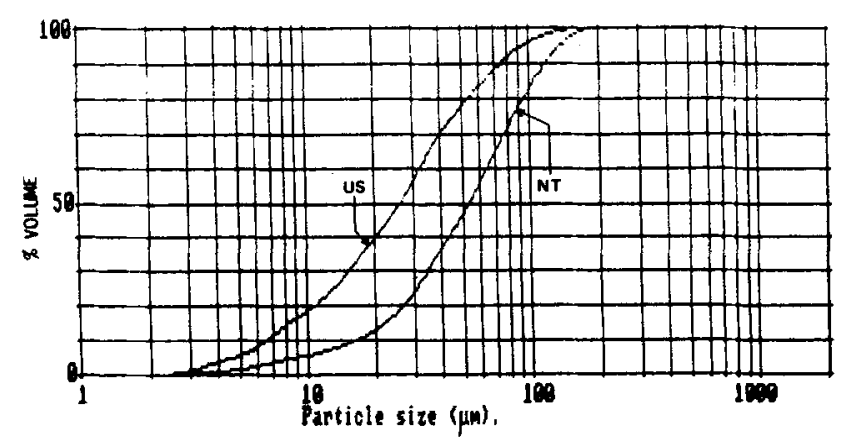

(a)

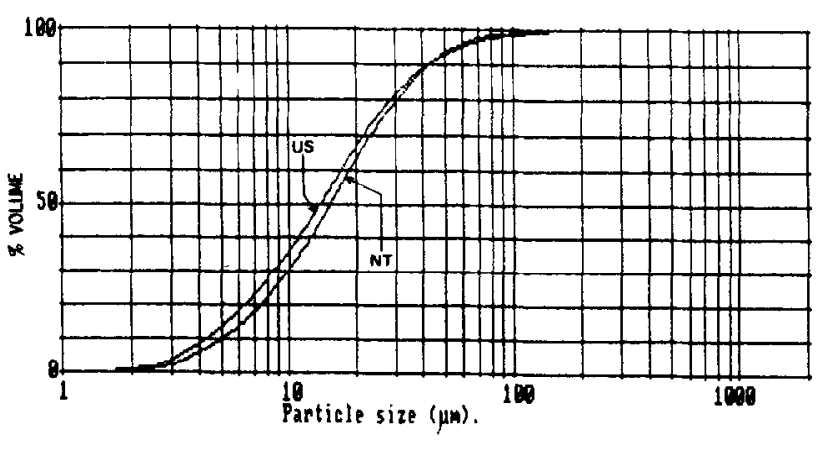

(b)

Figure 1 Volume cumulative undersize distributions given by the Malvern for Avicel (a) and Sigmacell (b) without (NT) and with (US) ultrasonic agitation

\section{Enzymatic fragmentation and stabilization of the particles}

Walker et al. ${ }^{10,27}$ studied the fragmentation of Avicel particles by purified enzymes from the Thermomonospora fusca and $T$. reesei. They found that the different proteins exhibit different fragmentation effectiveness. This is likely related to differences in the core binding domain (CBD) since, as shown by Din et al. ${ }^{28}$ purified CBDs are able to disrupt the structure of Ramie fibers.

The fragmentation of Avicel reported by Walker et al. ${ }^{27}$ was analyzed in this work. After $0.5 \mathrm{~h}$ of enzymatic activity (Ez1), the $d_{50}$ of Avicel drops by approximately half. These values are lower than those obtained after ultrasonic agitation (Table I).

As discussed by Rabinovitch (cited by Klyosov ${ }^{29}$ ), the dispersion of cellulose could result from either hydrolytic or mechanical action. The latter would be induced by the adsorption of the cellulases to disturbances of the crystalline structure of cellulose, followed by their penetration into the interfibrillar space. The enzyme concentration on the walls of pores and cavities of cellulose would increase the mechanical pressure and allow the penetration of water into the crevices which leads to the breaking of hydrogen bonds between the cellulose molecules.

In the present study, we tentatively explain the fragmenting and stabilizing effect of cellulases on Avicel particles on the basis of the DLVO theory and suggest that this is a phenomenon similar to that of defibrillation observed with other celluloses. In order to calculate the energy of the electrical interaction, $V_{\mathrm{R}}$, the zeta potential of the two types of cellulose was determined. The results obtained with Avicel for different $\mathrm{pH}$ values and ionic strength and for both US and Ezl samples are shown in Figure 3. As can be seen, the overall electrical charge of the US particles is always negative under the present experimental conditions. The zeta potential is less negative at lower $\mathrm{pH}$ values. On the other hand, the particles with adsorbed protein have zeta potential values of the same order of magnitude as those of the US sample at neutral and alkaline media. At $\mathrm{pH}$ values below approximately 3.5 , they become positively charged. This is because the main $T$. reese $i$ cellulases have acidic isoelectric points. CBH I, which amounts to about $60 \%$ of this cellulolytic system, has a pI between 3.6-4.1. Similar results were found for Sigmacell.

The calculation of the net energy of interaction for Avicel US particles as a function of the distance $d$ are shown in Figure 4. Similar profiles were obtained for Sigmacell. The attractive energy was lower than that calculated for Avicel for the same $d$ (probably due to the smaller particle size of Sigmacell). For the particles with adsorbed enzyme where $V_{\mathrm{T}}=V_{\mathrm{R}}$, the net energy increases when the particles get close to each other. On the contrary, at distances smaller than $5 \mathrm{~nm}$, an attraction exists in the sonicated particles. The DLVO theory therefore predicts a stabilizing effect of the particles by the adsorbed proteins.

In the case of Avicel, this prediction is in good agreement with the results of the obtained optical density attenu-

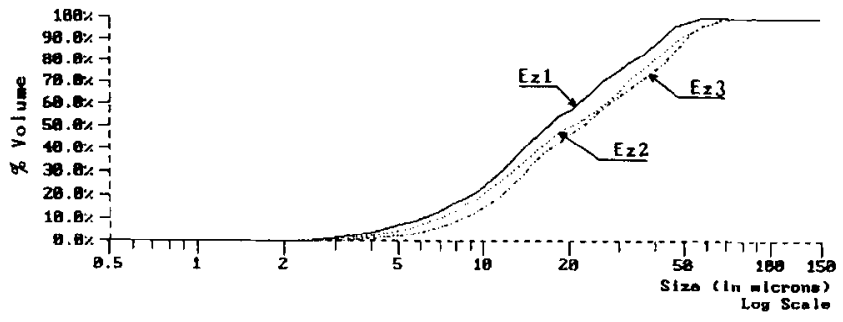

(a)

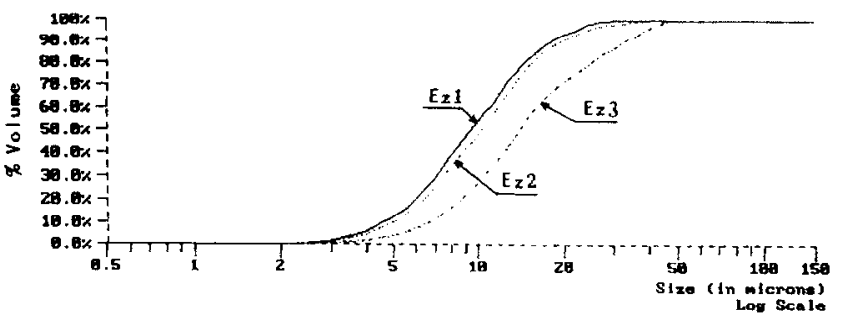

(b)

Figure 2 Influence of incubation time on particle size distribution of Avicel (a) and Sigmacell (b) given by the Galai 


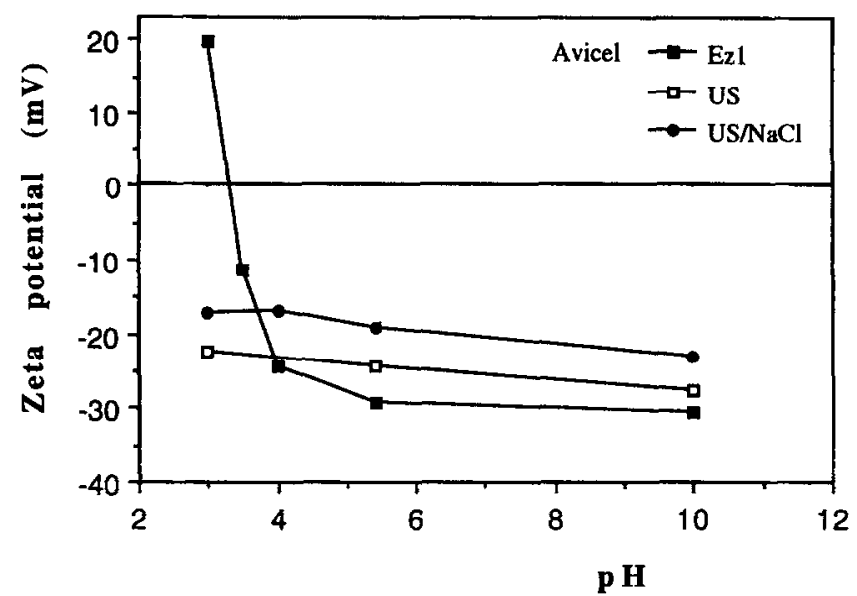

Figure 3 Zeta potential values of Avicel particles pretreated by ultrasonic agitation (US), enzymatic digestion (Ez1), or sonicated in $0.01 \mathrm{M} \mathrm{NaCl}$ (US/ $\mathrm{NaCl}$ )

ation curves whose slope is directly related to sedimentation velocity which in turn is related to particle (or aggregate) size. These results are displayed in Figure 5 and show that Avicel particles fragmented by enzymatic action (Ez1) sediment rather slower than those dispersed by ultrasonic agitation (US); moreover, it can be seen that both the enzyme and the ultrasound induce a similar increase in the initial optical density of the Avicel suspensions owing to the increase in the number of suspended particles. On the other hand, much smaller deviations were found for the optical attenuation curves of US and Ez1 samples of Sigmacell. It must be stressed that besides the DLVO forces, other forces are involved which contribute to particle stabilization. ${ }^{21}$ For instance, steric effects can greatly enhance the stability; moreover, the adsorbed proteins have large amounts of bound water which take part in particle interaction by hindering the approach of the particles. A different wettability is likely the reason why Sigmacell and Avicel display dissimilar optical density attenuation curves (Figure 5). Indeed, while Avicel particles aggregate, thereby increasing the sedimentation rate, Sigmacell particles do not aggregate probably because they have a lower crystallinity ( $79 \%$ for Sigmacell, $91 \%$ for Avicel). ${ }^{17}$ Sigmacell particles are more hydrated and stable in aqueous medium; thus, small differences in the crystallinity index seem to correspond to quite significant differences in surface properties such as the aggregation ability.

Additional experiments were performed with the Avicel US samples after addition of $0.05 \mathrm{M} \mathrm{NaCl}$ in order to study the effect of increasing ionic strength (Figure 5). A considerable increase in the sedimentation rate was found for Avicel which is in agreement with the DLVO theory. On the contrary for Sigmacell, no significant increase in the sedimentation rate was detected after addition of $\mathrm{NaCl}$.

Confirmation of these results was obtained using the Galai to measure the particle (or aggregate) size of these samples. In fact, larger particle sizes were obtained after addition of $\mathrm{NaCl}$ to the US samples of Avicel ( $d_{50}$ changed from 20 to $35 \mu \mathrm{m}$ ). No appreciable changes were detected for Sigmacell.

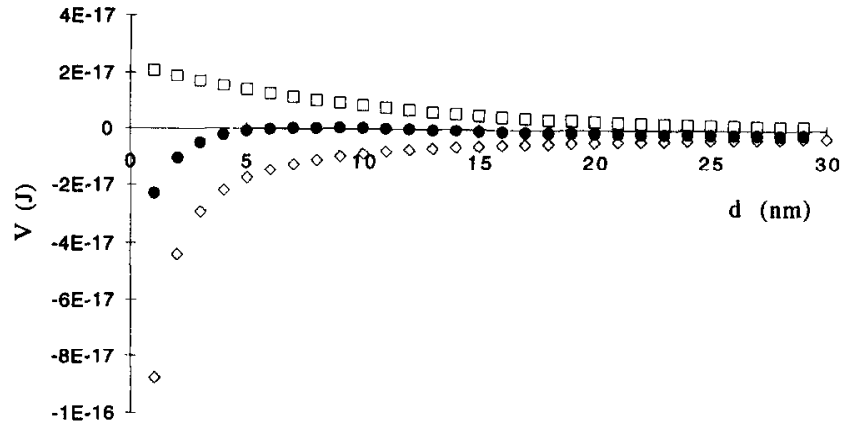

Figure 4 Repulsive $\left(V_{A}, \square\right)$, attractive $\left(V_{A} \diamond\right)$, and net energy

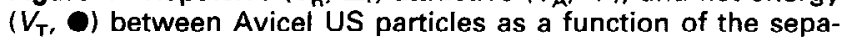
rating distance

These results suggest that, at least in the case of Avicel, the cellulose fragmentation by cellulases implies a physical stabilization of the particles by the adsorbed proteins. The mechanism of stabilization does not seem to be dependent on electrical interactions. As a matter of fact, the action of hydrophilic colloids in stabilizing hydrophobic colloids was recognized earlier by Freundlich (cited by Parfitt ${ }^{30}$ ) as not dependent on the enhancement of the charge of the hydro-
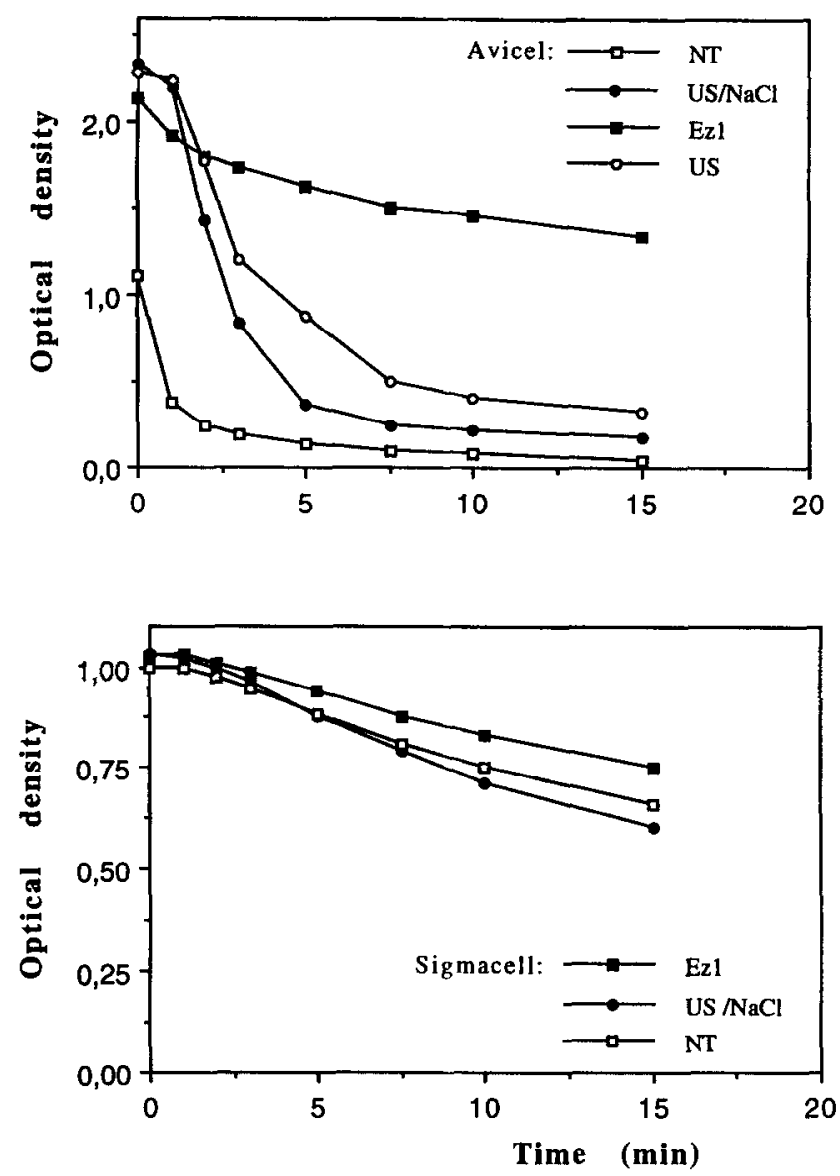

Figure 5 Optical density attenuation curves of the following Avicel and Sigmacell suspensions: nontreated (NT), sonicated (US), sonicated in $0.05 \mathrm{M} \mathrm{NaCl}$ (US/NaCl), and enzymatically treated (Ez1) 
phobic colloid, but rather on the strength of the adsorbed polymers and the way they are anchored to the particles.

The underlying mechanism for the enzymatic stabilization of cellulose probably requires a high surface coverage of the particles by the enzyme, which by surface diffusion, eventually would tear apart fibers attracted by van der Walls forces in the more crystalline areas.

\section{References}

1. Doelker, E., Guruy, R., Schurz, J., Janosi, A., and Matin, N. Degrees of crystallinity and polymerisation of modified cellulose powders. Powder Technol. 1987, 52, 207-213

2. Hsu, J.-C. and Penner. M. H. Preparation and utilization of cellulose substrates regenerated after treatment with hydrochloric acid. J. Agric. Food Chem. 1991, 39, 1444-1447

3. Gregory, J. The role of colloid interactions in solid-liquid separation. Water Sci. Technol. 1993, 27, 1-17

4. Stratton, R. A. and Swanson, J. W. Electrokinetics in papermaking. TAPPI 1981, 64, 79-83

5. Viikari, L., Kantelinen, A., Sundquist, J., and Linku, M. Xylanases in the bleaching: From an idea to the industry. FEMS Microbiol. Rev. 1994, 13, 335-350

6. Woodward. J., Affholter, K. A., Noles, K. K., Troy, N. T., and Gaslightwala, S. F. Does cellobiohydrolase II core disperse cellulose macrofibrils? Enzyme Microb. Technol. 1992, 14, 625-630

7. Woodward, J., Stephan, L. M., Koran, L. J., Wong, K. K. Y., and Saddler, J. N. Enzymatic separation of high-quality uninked pulp fibers from recycled newspaper. Bio/Technology 1994, 12, 905-908

8. Tyndall, R. M. Improving the softness and surface appearance of cotton fabrics and garments by treatment with cellulase enzymes. Text. Chem. 1992, 24, 23-26

9. Ramos, L. P., Nazhad, M. M., and Saddler, J. N. Effect of enzymatic hydrolysis on the morphology and fine structure of pretreated cellulosic residues. Enzyme Microb. Technol. 1993, 15, 821-831

10. Walker, L. P., Wilson, D. B., and Irwin, D. C. Measuring fragmentation of cellulose by Thermomonospora fusca cellulase. Enzyme Microb. Technol. 1990, 12, 378-386

11. Schell, D. J., Hinman, N. D., Grohmann, K., and Mohagheghi, A. Changes in physical and chemical properties of pretreated wheat straw during hydrolysis with cellulase. Biotechnol. Lett. 1989, 11, 745-748

12. Hayashida, S. and Mo. K. Production and characteristics of Aviceldisintegrating endoglucanase from a protease-negative Hurnicolu grisea var. thermoidea mutant. Appl. Environm. Microbiol. 1986, 51, 1041-1046

13. Halliwell, G. Hydrolysis of fibrous cotton and reprecipitated cellulose by cellulolytic enzymes from soil micro-organisms. Biochem. $J$. $1964,95,270-000$
14. Wood, T. M. Cellulolytic enzyme system of Trichoderma koningii. Biochem. J. 1968, 109, 217-227

15. Chanzy, H., Henrrissat, B., Vuong, R., and Schulein, M. The action of 1,4- $\beta$-D-glucan cellobiohydrolase on Valonia cellulose microcrystals. FEBS Lett. 1983, 153, 113-117

16. Ek, R., Alderborn, G., and Nystrom, C. Particle analysis of microcrystalline cellulose: Differentiation between individual particles and their agglomerates. Int. J. Pharm. 1994, 111, 43-50

17. Gama, F. M., Faro, C. J., Teixeira, J. A., and Mota, M. New methodology for the characterization of endoglucanase activity and its application on the Trichoderma longibrachiatum cellulolytic complex. Enzyme Microb. Technol. 1993, 15, 57-61

18. Gama, F. M., Teixeira, J. A., and Mota, M. Cellulose morphology and enzymatic reactivity: A modified solute exclusion technique. Biotechnol. Bioeng. 1994, 43, 381-387

19. Aharonson, E. P., Karasikov, N., Rojtberg, M., and Shamir, J. Galai CIS-1. A novel approach to aerosol particle size analysis. J. Aerosol Sci. 1986, 17, 530-536

20. Allen, H. Particle Size Measurement. Chapman and Hall, London, 1990, 455-479

21. Barth, H. Moderm Methods of Particle Size Analysis. Wiley, New York. 1984. 135-170

22. Hiemenz, P. C. Principles of Colloid and Surface Chemistry. Marcel Oekker, New York, 1986, 751

23. Blomberg, E., Claesson, P. M., Frobery, J. C., and Tilton, R. D. Interaction between adsorbed layers of lysozyme studied with the surface force technique. Langmuir 1994, 10, 2325-2334

24. Ferreira, P. J., Rasteiro, M. G., and Figueiredo, M. M. Influence of shape on particle size analysis. Particulate Sci. Technol. 1993, 11, 199-206

25. Seville, J. P. K., Coury, J. R., Ghadiri, M., and Clift, R. Comparison of techniques for measuring the size of fine non-spherical particles. Part. Charact. 1984, 1, 45-52

26. Nystrom, C., Malmqvist, K., and Alex, W. Comparison of permeametry and photometry for measurement of surface area of powders. Acta Pharm. Suec. 1977, 14, 497-504

27. Walker, L. P., Wilson, D. B., Irwin, D. C., McQuire. C., and Price, $\mathrm{M}$. Fragmentation of cellulose by the major Thermomonospora fusca cellulases, Trichoderma reesei $\mathrm{CBHI}$. and their mixtures. Biotechnol. Bioeng. 1992, 40, 1019-1026

28. Din, N., Gilkes, N. R., Tekant. B., Miller, R. C.. Warren. R. A. J.. and Kilburn, D. G. Non-hydrolytic disruption of cellulose fibers by the binding domain of a bacterial cellulase. Bio/Technology 1991, 9, $1096-1099$

29. Klyosov, A. A. Trends in biochemistry and enzymology of cellulose degradation. Biochemistry 1990, 29, 10577-10585

30. Parfitt, G. D. and Peacock, J. Stability of colloidal dispersions in nonaqueous media. In: Surface and Colloid Science (Matijevic. E.. Ed.). Plenum Press. New York, 1978. 163-226 